\title{
Population-based normative data for the Scoliosis Research Society 22r questionnaire in adolescents and adults, including a comparison with EQ-5D
}

\author{
Elias Diarbakerli $^{1,2} \cdot$ Anna Grauers ${ }^{1,3} \cdot$ Paul Gerdhem $^{1,2}$
}

Received: 12 February 2016/Revised: 20 September 2016/Accepted: 28 October 2016/Published online: 10 November 2016

(C) The Author(s) 2016. This article is published with open access at Springerlink.com

\begin{abstract}
Purpose The Scoliosis Research Society (SRS) 22r questionnaire is a widely used instrument. To estimate the disordeŕs impact on quality of life and to gain knowledge about treatment effects, normative values are needed.

Methods Individuals were randomly invited from the general population. 272 individuals (145 females) answered the SRS-22r and EuroQol 5-dimensions (EQ-5D) questionnaires and stratified according to sex and age; $\leq 19$ years $(n=61), 20-39$ years $(n=66), 40-59$ years $(n=84)$ and $\geq 60$ years $(n=61)$. The correlation between SRS-22r and EQ-5D were analyzed.

Results There were modest variations in mean SRS-22r scores (ranging between 4.3 and 4.7). EQ-5D followed the same pattern. The correlation between the SRS-22r was $0.62(p=0.001)$ and $0.61(p<0.001)$ for the EQ-5D UK tariff and EQ-5D Swedish tariff, respectively.

Conclusion We provide the first SRS-22r normative data for adolescents and adults overall. We found a good correlation between SRS-22r and EQ-5D in individuals without spinal deformity.
\end{abstract}

Electronic supplementary material The online version of this article (doi:10.1007/s00586-016-4854-0) contains supplementary material, which is available to authorized users.

Elias Diarbakerli

elias.diarbakerli@karolinska.se

1 Department of Clinical Sciences, Intervention and Technology (CLINTEC), Karolinska Institutet, K54, 14186 Stockholm, Sweden

2 Department of Orthopaedics, Karolinska University Hospital, Stockholm, Sweden

3 Department of Orthopaedics, Sundsvall and Härnösand County Hospital, Sundsvall, Sweden
Keywords SRS-22r · Normative · EQ-5D · Idiopathic . Scoliosis

\section{Introduction}

Quality of life in individuals with scoliosis has been investigated with various instruments [1-3]. Today, the Scoliosis Research Society (SRS) questionnaire, in its current SRS-22r version, is probably one of the most widely used scoliosis specific instruments. This questionnaire was originally intended to provide valid and reliable measures of quality of life in patients with scoliosis [4]. However, access to normative data is of interest when estimating the impact of spinal deformity on quality of life, and the effect of treatment. In addition, normative data might give information on specific domains that differ between individuals with and without spinal deformity, possibly of importance to address during treatment.

SRS-22r data for non-scoliotic individuals are lacking. Until now, studies have presented normative values for the earlier version, SRS-22, in adolescents in the United States $[5,6]$ but not for the SRS-22r. Normative data for adults have also been presented for SRS-22 [7] and SRS-30 [8], but not for this version, SRS-22r.

In addition, it is essential to have normative data that correspond to the cultural context in which the instrument is used. Demographic factors such as sex, household income and ethnicity may have a direct influence on perceived quality of life $[6,9]$. We find it of utmost importance to compare the disease specific instrument to a generic instrument to increase generalizability of the data.

EuroQol 5-dimensions (EQ-5D) is one of the most widely used generic instrument for measuring quality of life and reference values exist from a population-based 
study [10]. Both the SRS-22r and the EQ-5D are part of the patient reported outcome measures in the Swedish Spine register [11].

Hence, the aim of this population-based study was to present Swedish normative data on SRS-22r in both adults and adolescents, and to compare these with EQ-5D outcome measures. We also provide crude data for all individuals in this study, which will facilitate future use of these data by others.

\section{Materials and methods}

\section{Individuals}

Individuals were selected with the aim to represent all ages between 10 and 69 years with an equal sex and age distribution. Those aged 16 years and older were randomly selected from the Swedish population register and individuals younger than 16 years were randomly selected from the Stockholm County population register. We initially invited 4 females and 4 males in each age group. The response rate for those aged 18 and above was $51 \%$, and for those aged 17 and below 11\%. A flow chart of the recruitment process is shown in Fig. 1. The individuals were not examined. Six individuals reported being radiographed due to scoliosis and were excluded from the analyses. The final study cohort consisted of 272 individuals and 26 out of $272(10 \%)$ were born in another country than Sweden.

\section{Questionnaires}

SRS-22r is a scoliosis specific instrument developed aiming to measure quality of life outcomes in scoliosis patients. It has been translated to Swedish and has been

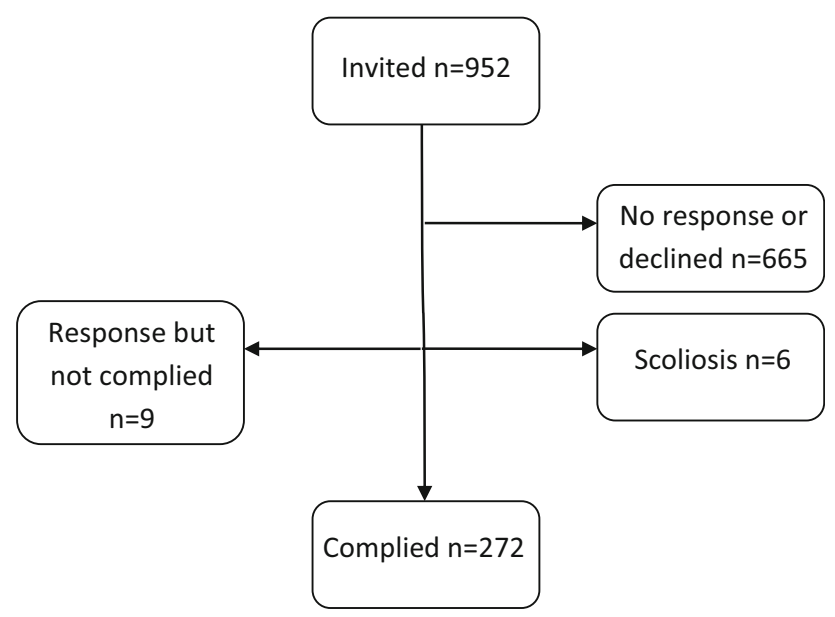

Fig. 1 An illustration of the recruitment process tested regarding reliability and validity [12]. The questionnaire comprises 22 questions distributed over five domains (function, pain, self-image, mental health and satisfaction) and each domain has five answers (one being the worst and five the best). It is possible to derive an index for each domain, and further, a total index for all domains. The satisfaction domain cannot be used for those who have not been treated for scoliosis. Accordingly, we present the SRS-22r subscore, excluding the satisfaction domain, in this study. The subscore could not be obtained if scores on more than two domains were missing.

EQ-5D is a general health instrument in which individuals assess their health in five dimensions including mobility, self-care, usual activities, pain/discomfort and anxiety/depression [13, 14]. In this study, we used the 3 level version (EQ-5D-3L) where the individual chooses between three levels based on how well he/she can manage each dimension. The answers have been translated to an index based on a tariff representing the societal perspective on health. In this study, we used the British tariff [15] and a recently published Swedish tariff [16]. Using the UK tariff, it is possible to obtain an index between -0.59 (worst) and 1.0 (best). Using the Swedish tariff, it is possible to obtain values between 0.34 (worst) and 0.97 (best).

\section{Other data}

In addition, our survey included general questions on health, employment status, anthropometrics, spinal problems, presence of chronic disorders (heart disorders, neurological diseases, diabetes, asthma, lung disorders, epilepsy or other chronic disorder, which could be specified), working situation and medications.

\section{Data management}

Data are presented as mean ( $95 \%$ confidence interval; $\mathrm{CI}$ ) or number (percentage). The individuals were stratified according to sex and age; $\leq 19$ years, 20-39 years, 40-59 years and 60 years or older. A comparison was done using analysis of covariance, adjusted for age and sex, to compare SRS-22r subscore and EQ-5D indexes between individuals born in and outside Sweden. The Spearman test was used to analyze correlations between the SRS-22r subscore and the EQ-5D index, and between domains that were considered similar: the pain domains in both questionnaires; the mobility and the self-care domain in the EQ$5 \mathrm{D}$ and the function domain in the SRS-22r; the anxiety domain in EQ-5D and the mental health domain in SRS22r. Variation of the SRS-22r subscore with age was graphically assessed. To allow comparisons with previously published data on SRS22 we converted the SRS22r 
subscore to SRS22 according to Lai et al. [17]; SRS22 subscore $=($ SRS22r subscore-0.040)/1.009.

\section{Ethical consent}

Written informed consent was obtained from all study participants, and from their legal guardians if the individual was under the age of 16 years. The ethical review board in Stockholm approved all parts of the study (Dnr 2012/17231/4).

\section{Results}

Descriptive data are presented in Table 1. More females than males were included in all but the oldest age group. As expected, chronic disorders, back and neck problems were most frequent in the two oldest groups.

There were, in general, small differences in the SRS-22r between sexes and age groups. The mean SRS-22r subscore varied between 4.3 (women aged 40-59) and 4.7 (men aged $\leq 19$ ), (Table 2; Fig. 2). Males tended to have higher SRS-22r subscore than females in the youngest age group, but not in the other age groups, (Table 2). The EQ$5 \mathrm{D}$ index, based on the UK tariff, tended to be lower with higher age, (Table 2).

The separate domain scores for SRS-22r are presented in Table 3. In general, the mean domain scores tended to be lower in the two oldest age groups, with an exception for the mental health domain that was higher among those aged $\geq 60$ years than in those aged $40-59$ years.
The separate dimensions in EQ-5D are presented in Table 4. The number of individuals with problems increased with age in the EQ-5D domains mobility, usual activities and pain, but decreased for anxiety in those aged $\geq 60$ years compared to those aged 40-59 years.

Spearman's correlation coefficient was $0.62(p<0.001)$ when comparing the SRS-22r subscore and the EQ-5D index (UK tariff and $0.61(p<0.001)$ when comparing the SRS-22r subscore and the EQ-5D index Swedish tariff). When separate domains were compared in the two questionnaires, the Spearman correlation coefficient was -0.61 $(p<0.001)$ for the pain domains, $-0.24(p<0.001)$ for the function domain in the SRS-22r and the mobility domain in the EQ-5D, $-0.66(p=0.28)$ for the function domain in SRS-22r and the self-care domain in the EQ-5D, and $-0.55(p<0.001)$ for the mental health domain in the SRS-22r and the anxiety domain in the EQ-5D.

Individual data for the whole cohort are presented in Supplementary Table 1, including the SRS-22r subscore, the calculated SRS-22 subscore and the EQ-5D indexes.

No differences were seen in EQ-5D indexes when comparing individuals born in or outside Sweden (all $p \geq 0.46$ ). Mean SRS-22r subscore for individuals born in Sweden was 4.5 (4.4-4.6) and for individuals born outside Sweden 4.3 (4.2-4.5) ( $p=0.005$; adjusted for age and sex).

\section{Discussion}

This population-based study presents normative data for the SRS-22r and the EQ-5D in adults and adolescents of various ages. Age and sex specific variations in SRS-22r

Table 1 Descriptive data in all age groups. Data is presented as mean (CI 95\%) or number (\%)

\begin{tabular}{lllll}
\hline & Age $\leq 19(n=61)$ & Age 20-39 $(n=66)$ & Age 40-59 $(n=84)$ & Age $\geq 60(n=61)$ \\
\hline Females & $38(62 \%)$ & $39(59 \%)$ & $43(51 \%)$ & $25(41 \%)$ \\
Age $(y)$ & $14.5(14.0-15.1)$ & $29.9(28.4-31.3)$ & $48.6(47.3-49.9)$ & $65.0(64.2-65.8)$ \\
Height $(\mathrm{m})$ & $1.65(1.62-1.68)$ & $1.73(1.71-1.76)$ & $1.74(1.72-1.76)$ & $1.74(1.72-1.77)$ \\
Weight $(\mathrm{kg})$ & $56(52-59)$ & $78(73-82)$ & $81(77-84)$ & $79(74-83)$ \\
Smokers & $1(2 \%)$ & $9(14 \%)$ & $10(12 \%)$ & $5(8 \%)$ \\
Studying & $60(98 \%)$ & $13(20 \%)$ & $2(2 \%)$ & $0(0 \%)$ \\
Gainfully employed & $2(3 \%)$ & $44(67 \%)$ & $78(93 \%)$ & $26(43 \%)$ \\
Sporting activities & $37(61 \%)$ & $33(50 \%)$ & $35(42 \%)$ & $16(26 \%)$ \\
Back problems & $11(18 \%)$ & $18(27 \%)$ & $32(38 \%)$ & $20(33 \%)$ \\
Neck problems & $10(16 \%)$ & $6(9 \%)$ & $24(29 \%)$ & $16(26 \%)$ \\
Chronic disorder & $6(10 \%)$ & $11(17 \%)$ & $25(30 \%)$ & $24(39 \%)$ \\
Regular medication & $8(13 \%)$ & $22(33 \%)$ & $36(43 \%)$ & $41(67 \%)$ \\
\hline
\end{tabular}

$m$ metres, $k g$ kilograms, $y$ years 
Table 2 Mean (CI 95\%) SRS-22r subscore index and EQ-5D index for all age groups, including men and women

\begin{tabular}{lllll}
\hline & Age $\leq 19(n=61)$ & Age 20-39 $(n=66)$ & Age 40-59 $(n=84)$ & Age $\geq 60(n=61)$ \\
\hline SRS-22r subscore index-all & $4.7(4.6-4.8)$ & $4.5(4.4-4.6)$ & $4.4(4.2-4.5)$ & $4.5(4.3-4.6)$ \\
SRS-22r subscore index-women & $4.6(4.5-4.7)$ & $4.5(4.4-4.6)$ & $4.3(4.1-4.5)$ & $4.4(4.2-4.6)$ \\
SRS-22r subscore index-men & $4.7(4.6-4.8)$ & $4.5(4.3-4.6)$ & $4.4(4.3-4.6)$ & $4.5(4.3-4.6)$ \\
EQ-5D index-all-UK tariff [15] & $0.93(0.89-0.97)$ & $0.90(0.86-0.93)$ & $0.87(0.83-0.91)$ & $0.87(0.84-0.91)$ \\
EQ-5D index-women-UK tariff [15] & $0.91(0.85-0.97)$ & $0.91(0.87-0.94)$ & $0.86(0.80-0.91)$ & $0.84(0.78-0.90)$ \\
EQ-5D index-men-UK tariff [15] & $0.97(0.93-1.0)$ & $0.88(0.81-0.96)$ & $0.89(0.83-0.95)$ & $0.90(0.86-0.94)$ \\
EQ-5D index-all-Swedish tariff [16] & $0.95(0.93-0.96)$ & $0.94(0.92-0.95)$ & $0.93(0.91-0.94)$ & $0.93(0.91-0.94)$ \\
EQ-5D index-women-Swedish tariff [16] & $0.94(0.92-0.96)$ & $0.94(0.93-0.96)$ & $0.92(0.89-0.94)$ & $0.92(0.89-0.94)$ \\
EQ-5D index-men-Swedish tariff [16] & $0.96(0.94-0.97)$ & $0.93(0.90-0.96)$ & $0.94(0.92-0.96)$ & $0.94(0.92-0.96)$ \\
\hline
\end{tabular}

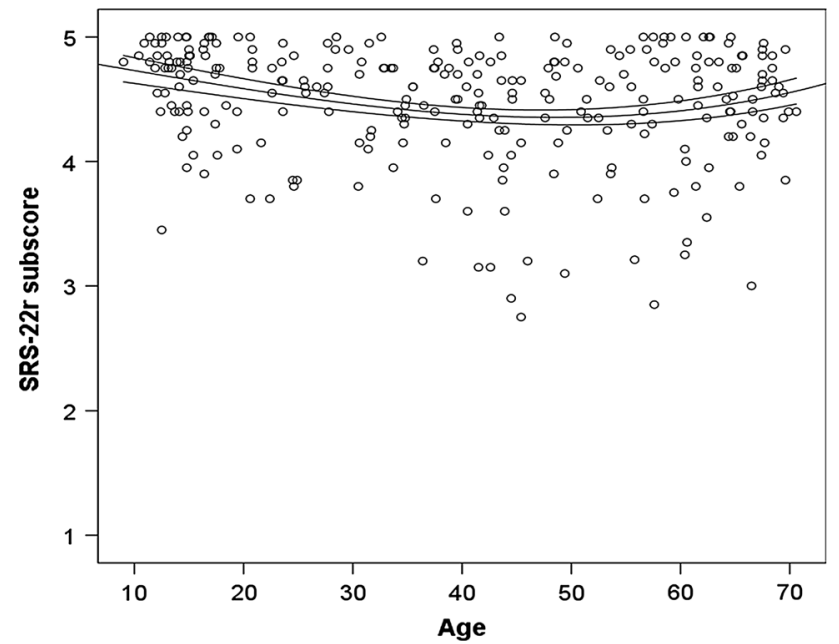

Fig. 2 The association between the SRS-22r subscore and age. The best fit was obtained with a cubic-spline curve. The mean and the $95 \%$ confidence intervals are depicted were small. We found a moderate to good correlation between the SRS-22r and EQ-5D questionnaires. Data from this study will enable comparisons with individuals having spinal deformities.

A previous study from the United States, presenting population-based data for SRS-22 in 34 adults (mean age 46 years), showed slightly lower scores on all domains compared to the adults in our study [7].

Previous studies reporting SRS-22 outcomes in healthy adolescents in the United States have shown slightly lower scores on all domains compared to those aged $\leq 19$ years in this study [5, 6]. Even though converting SRS-22r scores to SRS22 values according to Lai et al. [17], we still obtained higher scores than in the previous studies (Supplementary Table 1). However, converting SRS-22r values in controls according to Lai et al. might not be completely accurate since the sample used to construct the conversion algorithm was based on 121 surgically treated individuals, aged 14-34 years [17].

Table 3 Mean (CI 95\%) SRS-22r domain scores in all age groups, stratified on sex

\begin{tabular}{lllll}
\hline & Age $\leq 19(n=61)$ & Age 20-39 $(n=66)$ & Age 40-59 $(n=84)$ & Age $\geq 60(n=61)$ \\
\hline Function-all & $4.8(4.7-4.9)$ & $4.7(4.6-4.8)$ & $4.6(4.4-4.7)$ & $4.5(4.4-4.6)$ \\
Function-women & $4.8(4.6-4.9)$ & $4.8(4.7-4.8)$ & $4.5(4.3-4.7)$ & $4.5(4.3-4.8)$ \\
Function-men & $4.8(4.6-4.9)$ & $4.7(4.6-4.9)$ & $4.6(4.5-4.8)$ & $4.5(4.4-4.7)$ \\
Pain-all & $4.9(4.8-4.9)$ & $4.6(4.5-4.8)$ & $4.4(4.2-4.6)$ & $4.5(4.3-4.7)$ \\
Pain-women & $4.8(4.7-4.9)$ & $4.7(4.5-4.8)$ & $4.3(4.1-4.6)$ & $4.5(4.2-4.9)$ \\
Pain-men & $4.9(4.8-5.0)$ & $4.6(4.4-4.8)$ & $4.5(4.3-4.7)$ & $4.5(4.2-4.7)$ \\
Self-image-all & $4.7(4.6-4.8)$ & $4.5(4.4-4.7)$ & $4.4(4.2-4.5)$ & $4.4(4.3-4.5)$ \\
Self-image-women & $4.8(4.7-4.9)$ & $4.6(4.5-4.7)$ & $4.3(4.2-4.5)$ & $4.4(4.2-4.6)$ \\
Self-image-men & $4.6(4.4-4.8)$ & $4.5(4.2-4.7)$ & $4.4(4.2-4.6)$ & $4.4(4.2-4.6)$ \\
Mental health-all & $4.3(4.1-4.5)$ & $4.1(3.9-4.3)$ & $4.1(3.9-4.2)$ & $4.4(4.3-4.6)$ \\
Mental health-women & $4.1(3.9-4.4)$ & $4.1(3.9-4.3)$ & $4.0(3.7-4.2)$ & $4.4(4.1-4.7)$ \\
Mental health-men & $4.5(4.3-4.7)$ & $4.1(3.8-4.4)$ & $4.2(4.0-4.5)$ & $4.5(4.3-4.7)$ \\
\hline
\end{tabular}




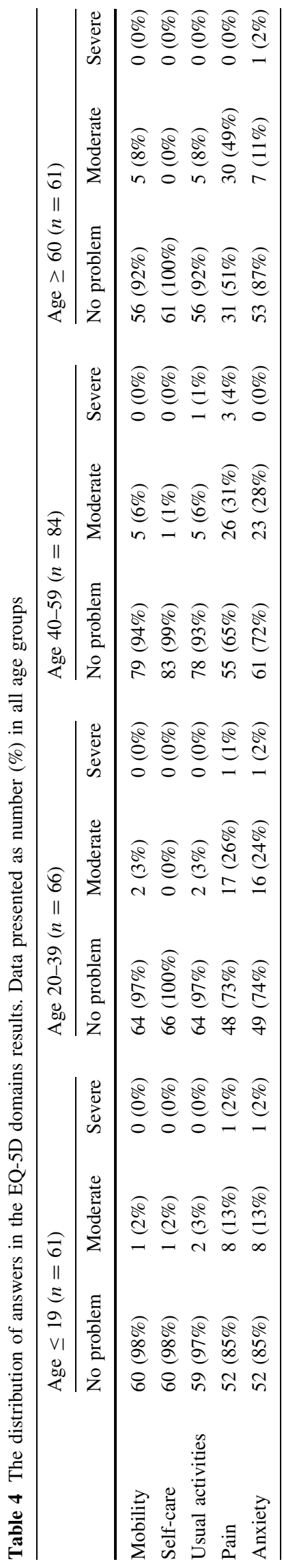

Other explanations for the differing results between the previous and the present study might be differences in sample size, and sampling methods [5-7]. Both the previous studies concerning adolescents $[5,6]$ were performed in schools with anonymous questionnaires. Another study on adults only included 34 individuals [7]. Our study was population based and included adolescents and adults from different areas in Sweden, even though the youngest individuals were recruited from Stockholm County.

Other reasons to be considered for these differences are cultural aspects, including differences due to translation of the questionnaire, different levels of physical activity, and different perceptions of pain and mental health [18]. Even when using the same language version, differences between healthy children in different countries have been reported [9] indicating the need for country-specific normative data.

There were only minor age and sex related variations in SRS-22r and EQ-5D in this study. Individuals in the age group 40-59 reported slightly lower scores than those in the oldest age group. This is not unique for the SRS-22r. Similar findings have been described for the EQ-5D, and especially for the anxiety domain which had a modest correlation to the mental health domain in the SRS-22r in this study [10]. In the adolescent age group, males had higher SRS-22r subscore than females, with the same tendency seen for EQ-5D in this and in other studies [10].

Previous studies on the SRS-22r, the SRS-22, and other questionnaires in young individuals have been inconsistent concerning sex differences $[5,6,9,10,19]$. We think it is advisable, if possible, to use sex specific data when normative data and patient data are compared. Furthermore, the importance of assessing appropriate outcome instruments when managing spinal disorders have been highlighted since there is a broad variety of instruments and tools measuring outcomes of spinal disorders and their treatments [20].

An obvious strength of this study is that it comprises data for males, females, adults and adolescents, providing a broad perspective of SRS-22r outcomes in individuals without a spinal deformity. Furthermore, the SRS-22r was compared and correlated to the generic EQ-5D instrument. We found moderate to good correlations between the two instruments and for the corresponding domains pain and mental health/anxiety.

Despite the similarities between the SRS-22r and EQ$5 \mathrm{D}$, correlations are not perfect. Therefore, the use of both disease specific and generic quality of life instruments is often advocated [21, 22]. Accordingly, both the SRS-22r and the EQ-5D are used as patient reported outcome measures in the Swedish Spine register (SweSpine) [11].

A major limitation of this study is the low response rate for the youngest individuals, challenging the representativeness of this study. Nevertheless, this population-based 
study consists of adults and adolescents regardless of geographic localization in Sweden. It has been suggested that representativeness might not be necessary other than that the controls should not have the studied trait [23]. In addition, comparisons with other Swedish populationbased cohorts regarding EQ-5D did not indicate any important differences [10]. Data on household income was not obtained and, therefore, not compared in the individuals.

We used the EQ-5D in the youngest individuals to be able to compare the results with adults, as suggested by others [21]. EQ-5D-Y is a version of EQ-5D specifically adopted for children. Compared to reported domain results for EQ-5D-Y in Swedish adolescents [24] we obtained similar results indicating representativeness of our sample.

Another limitation is the sample size. A larger sample size would have enabled more detailed studies of age and sex differences. Nevertheless, age and sex differences were fairly small and within or close to the suggested minimal clinical important differences for the SRS-22r in patients treated for scoliosis [25].

\section{Conclusion}

This study provides the first normative data for SRS-22r in Swedish adults and adolescents, including both males and females. The normative SRS-22r scores in this study are slightly higher than previously reported data on SRS-22, despite conversion to SRS-22 values, illustrating the need for country-specific normative values. In addition, this study showed a good correlation when comparing SRS-22r and EQ-5D in individuals without a spinal deformity. We consider our material to be useful as reference for future studies of individuals with spinal deformity. The presentation of crude data for all individuals in this study will ease future comparisons.

\section{Compliance with ethical standards}

Conflict of interest The authors have no conflict of interest to declare.

Source of funding This study was financially supported by funds from the regional agreement on medical training and clinical research (ALF) between Stockholm County Council and Karolinska Institutet, the Karolinska Institutet research funds, the Swedish Research Council (K2013-99X-22268-01-3), and the Swedish Society of Spinal Surgeons.

Open Access This article is distributed under the terms of the Creative Commons Attribution 4.0 International License (http://crea tivecommons.org/licenses/by/4.0/), which permits unrestricted use, distribution, and reproduction in any medium, provided you give appropriate credit to the original author(s) and the source, provide a link to the Creative Commons license, and indicate if changes were made.

\section{References}

1. Weinstein SL, Dolan LA, Spratt KF, Peterson KK, Spoonamore MJ, Ponseti IV (2003) Health and function of patients with untreated idiopathic scoliosis: a 50-year natural history study. JAMA 289(5):559-567

2. Grauers A, Topalis C, Moller H, Normelli H, Karlsson M, Danielsson A, et al (2014) Prevalence of Back Problems in 1069 Adults With Idiopathic Scoliosis and 158 Adults Without Scoliosis. Spine

3. Danielsson AJ, Wiklund I, Pehrsson K, Nachemson AL (2001) Health-related quality of life in patients with adolescent idiopathic scoliosis: a matched follow-up at least 20 years after treatment with brace or surgery. Eur Spine J 10(4):278-288

4. Haher TR, Gorup JM, Shin TM, Homel P, Merola AA, Grogan DP et al (1999) Results of the Scoliosis Research Society instrument for evaluation of surgical outcome in adolescent idiopathic scoliosis. A multicenter study of 244 patients. Spine. 24(14):1435-1440

5. Daubs MD, Hung M, Neese A, Hon SD, Lawrence BD, Patel AA et al (2014) Scoliosis research society-22 results in 3052 healthy adolescents aged 10 to 19 years. Spine 39(10):826-832

6. Verma K, Lonner B, Hoashi JS, Lafage V, Dean L, Engel I et al (2010) Demographic factors affect Scoliosis Research Society-22 performance in healthy adolescents: a comparative baseline for adolescents with idiopathic scoliosis. Spine 35(24):2134-2139

7. Berven S, Deviren V, Demir-Deviren S, Hu SS, Bradford DS (2003) Studies in the modified Scoliosis Research Society Outcomes Instrument in adults: validation, reliability, and discriminatory capacity. Spine 28(18):2164-2169 (discussion 9)

8. Baldus C, Bridwell K, Harrast J, Shaffrey C, Ondra S, Lenke L et al (2011) The Scoliosis Research Society Health-Related Quality of Life (SRS-30) age-gender normative data: an analysis of 1346 adult subjects unaffected by scoliosis. Spine. 36(14):1154-1162

9. Verma K, Lonner B, Toombs CS, Ferrise P, Wright B, King AB et al (2014) International utilization of the SRS-22 instrument to assess outcomes in adolescent idiopathic scoliosis: what can we learn from a medical outreach group in Ghana? J Pediatr Orthop 34(5):503-508

10. Burstrom K, Johannesson M, Diderichsen F (2001) Swedish population health-related quality of life results using the EQ-5D. Qual Life Res 10(7):621-635

11. Ersberg A, Gerdhem P (2013) Pre- and postoperative quality of life in patients treated for scoliosis. Acta Orthopaedica. 84(6):537-543

12. Danielsson AJ, Romberg K (2013) Reliability and validity of the Swedish version of the Scoliosis Research Society-22 (SRS-22r) patient questionnaire for idiopathic scoliosis. Spine. 38(21):1875-1884

13. EuroQol Group (1990) EuroQol-a new facility for the measurement of health-related quality of life. Health Policy 16(3):199-208

14. Rabin R, de Charro F (2001) EQ-5D: a measure of health status from the EuroQol Group. Ann Med 33(5):337-343

15. Dolan P (1997) Modeling valuations for EuroQol health states. Med Care 35(11):1095-1108

16. Burstrom K, Sun S, Gerdtham UG, Henriksson M, Johannesson M, Levin LA et al (2014) Swedish experience-based value sets for EQ-5D health states. Qual Life Res 23(2):431-442 
17. Lai SM, Burton DC, Asher MA, Carlson BB (2011) Converting SRS-24, SRS-23, and SRS-22 to SRS-22r: establishing conversion equations using regression modeling. Spine 36(23):E1525E1533

18. Watanabe K, Lenke LG, Bridwell KH, Hasegawa K, Hirano T, Endo N et al (2007) Cross-cultural comparison of the Scoliosis Research Society Outcomes Instrument between American and Japanese idiopathic scoliosis patients: are there differences? Spine 32(24):2711-2714

19. Helseth S, Haraldstad K, Christophersen KA (2015) A crosssectional study of Health Related Quality of Life and body mass index in a Norwegian school sample (8-18 years): a comparison of child and parent perspectives. Health and Qual Life Outcomes 13:47

20. Vavken P, Ganal-Antonio AK, Quidde J, Shen FH, Chapman JR, Samartzis D (2015) Fundamentals of clinical outcomes assessment for spinal disorders: clinical outcome instruments and applications. Global Spine J 5(4):329-338
21. Noyes J, Edwards RT (2011) EQ-5D for the assessment of healthrelated quality of life and resource allocation in children: a systematic methodological review. Val Health 14(8):1117-1129

22. Protudjer JL, Jansson SA, Ostblom E, Arnlind MH, Bengtsson U, Dahlen SE et al (2015) Health-related quality of life in children with objectively diagnosed staple food allergy assessed with a disease-specific questionnaire. Acta Paediatr 104(10):1047-1054

23. Rothman KJ, Gallacher JE, Hatch EE (2013) Why representativeness should be avoided. Int J Epidemiol 42(4):1012-1014

24. Burstrom K, Bartonek A, Brostrom EW, Sun S, Egmar AC (2014) EQ-5D-Y as a health-related quality of life measure in children and adolescents with functional disability in Sweden: testing feasibility and validity. Acta Paediatr 103(4):426-435

25. Crawford C. H, 3rd, Glassman S. D, Bridwell K. H, Berven S. H, Carreon L. Y. The minimum clinically important difference in SRS-22R total score, appearance, activity and pain domains after surgical treatment of adult spinal deformity. Spine. 2015;40(6):377-81 